\title{
Innovation System Construction for China's Basic and Clinical Medicine
}

\author{
Fu Lei ${ }^{1}$, Yin Ling ${ }^{1}$, Zhu Manlu ${ }^{2}$, Ma Jie $^{2}$, Gao Runlin ${ }^{2}$ \\ 1. Chinese People's Liberation Army General Hospital, Beijing 100853, China \\ 2. Fu Wai Hospital, Chinese Academy of Medical Sciences, Beijing 100037, China
}

\begin{abstract}
This paper is a summary of China's Basic and Clinical Medicine Research and Innovation System, a subproject of the major advisory project titled the "Development Strategy for National Health Promotion and Medical and Health Undertakings in China" launched by the Chinese Academy of Engineering. This paper describes an in-depth analysis of the medical research management and system construction of major world powers and of the major obstacles that hinder innovation medical science and technology in China. It then puts forward a list of suggestions that include strengthening the top-level design of medical research, restructuring the medical research system, increasing research funding, improving the evaluation system, integrating shared resources in medical science and technology, and accelerating the translation of medical research achievements.
\end{abstract}

Keywords: national health; medical research; innovation system

\section{Introduction}

Medical technology is the cornerstone of the "Healthy China" strategy. In recent years, the Chinese government has significantly increased funding for public health, and medical research funding has also increased annually; as a result, China's medical science and technology has seen dramatic progress in innovation, research output, and the medical and health industry. However, China's medical research still displays some noticeable weaknesses such as inadequate innovation, poor research outcomes, weak pharmaceutical R\&D ability, and so on in the face of the national goal of "National One-stop Health" and the increasing demand for medical services.

In June of 2014, the Chinese Academy of Engineering launched the major consulting project "Development Strategy for National Health Promotion and Medical and Healthcare Undertakings in China." As one of its subprojects, "China's Basic and Clinical Medical Research and Innovation System" has attracted more than 30 academicians, experts, and scholars from the medical and health and science and technology management fields to jointly work on it. Eyeing the needs of the national health and medicine and health industry development strategy, they used various methods such as literature research, surveys, consultations, and seminars to conduct in-depth analysis on the medical research management and system construction of the major world powers and consulted nearly 100 experts on the major obstacle that restrains the innovation of China's medical science and technology, finally putting forward suggestions for innovation in China's medical research. This paper presents systematic consideration based on the research report and the latest trend of China's science and technology system reform.

\section{Analysis on advanced countries' medical research management and system building}

\subsection{Government-led planning and promotion of medical research}

Government-led planning and promotion of medical research is a popular practice for medicine in advanced countries. The

Received date: January 15, 2017; Revised date: February 28, 2017

Corresponding author: Gao Runlin, Fu Wai Hospital, Chinese Academy of Medical Sciences, Professor; Chinese Academy of Engineering, Academician. Major research field is cardiology. E-mail: gaorunlin@citmd.com

Funding program: CAE Advisory Project “Development Strategy for National Health Promotion and Medical and Healthcare Undertakings in China” (2014-ZD-06)

Chinese version: Strategic Study of CAE 2017, 19 (2): 072-078

Cited item: Fu Lei et al. Innovation System Construction of China's Basic and Clinical Medicine. Strategic Study of CAE, https://doi.org/10.15302/J-SSCAE-2017.02.012 
US, the UK, France, Germany, Australia, Japan, and India have developed distinctive medical research management systems based on their own science and technology situations. The US is the most prominent in this aspect among these countries.

The current US medical research management adopts a topdown national action. The National Institute of Health (NIH), a subsidiary of the Department of Health and Human Services, takes charge of medical research. As a combination of medical research and administration and owning 19 research institutes, 7 research centers, and one national library of medicine, the NIH is America's most important medical research base and the biggest medical research management body managing more than $90 \%$ of the federal government-led medical research funding, about 30 billion USD annually in recent years. It manages and regulates medical research through funding [1]. The NIH was given huge authority in the planning of research projects and allocation of funds and can appropriately pinpoint and plan key development areas through collecting and analyzing past project data and considering the current cutting-edge areas and current and future national needs.

Similar to the NIH, the UK Medical Research Council (MRC), French National Health and Medical Research Institute (Institut National de la Santé et de la Recherche Médicale, INSERM), Australian National Health and Medical Research Council (NHMRC), and Indian Council of Medical Research are also in charge of national medical research, developing the management policies and development strategies in the biomedical field and coordinating the relationship between research and medical service. In April of 2015, Japan's government established the Japan Agency for Medical Research and Development (AMED) and positioned it as the headquarters of Japanese medical research; therefore, AMED is also called the Japanese version of the NIH and aims to plan and manage medical research and to establish a complete medical R\&D system from basic research to practical application to become the leading medical service in the world.

This centralized management structure that can uniformly plan and promote medical research and a professional team-led research management mode ensure the leading edge and efficiency of medical research.

\subsection{Projects, resources, and services seamlessly integrate to form a complete medical innovation system covering administration, production, teaching, and research}

The countries advanced in medicine have successfully integrated different resources to build a complete research system that includes administration, production, teaching, and research elements such as basic research, $R \& D$, industrial promotion, and so on, thereby effectively improving the research quality and efficiency and realizing the quick application of medical research achievements.

The forum "Promoting Efficient and Effective Collaborations among Academia, Government and Industry" held by the NIH pools officials, experts, and professionals from governments, academia, businesses, and non-governmental organizations to discuss needs, issues, and problems in the process of medical research and biopharmaceutical industry development, brainstorming good solutions to faster transform research achievements into service capabilities [2]. As the medical regulator of the US federal government, the NIH reserves only a small part of its funds (about $10 \%$ of annual funding) for its own research institutes or centers and spends about $80 \%$ of the funds on other research institutes, including over 3000 universities, medical schools, and public or private research bodies across the US as well as on valuable projects worldwide. By funding interdisciplinary medical research, the NIH can effectively integrate basic and clinical medicine and can also receive feedback from experts in different fields through extensive cooperation, thus quickly solving the problems arising from the research.

In the UK, the National Clinical Research Network Alliance has effectively integrated the stakeholders of national clinical research, including public or private research funders, research institutes, the national health care service system, third-party mediation bodies, pharmaceutical companies, and research objects by pooling the resources to the utmost extent, greatly improving the efficiency of research and conversion. To strengthen the strategic cooperation and project coordination of life and health science research, INSERM, together with other research institutions in France and the International Association of University Presidents, established the French National Union of Life and Health Sciences in April of 2009, highlighting the research coordination work from the perspective of national strategy [3]. To promote cooperation between businesses and research institutions, the German government established a large medical high-tech park in the 1980s, supporting start-up high-tech pharmaceutical companies or helping new companies spin off from research institutions, thereby accelerating the flow, spread, and application of research achievements and knowledge among users and creators.

In the medical innovation system of advanced countries, the government or relevant authorities have effectively integrated the science and technology resources, research projects, and services as well as sound top-level design, macro guidance, solid infrastructure, and complete policy environment, greatly boosting innovation.

\subsection{Established a relatively complete and reasonable research evaluation system}

Research evaluation acts as a "lighthouse" in promoting and guiding research. The US is one of the earliest and most mature countries in developing and improving a medical research evaluation system, which it built based on peer reviews, thus improving the government's management and efficiency and ensuring 
the realization of the US national science and technology goal.

The NIH has established a rather complete and reasonable scientific evaluation system to guide research projects and evaluate the scientific and technological personnel [4-6]. The NIH adopted a two-step evaluation rule for competitive research projects, and the NIH evaluation center organizes and implements the rule. The preliminary evaluation is carried out by the evaluation group, which is made up of 16-20 non-governmental scientists who are leaders in their respective disciplines; the secondary evaluation is carried out by the national advisory committee, which is made up of 12-18 famous scientists from research institutes as well as public representatives who are interested in medicine or biomedicine. Only the projects going through the two-step evaluation can be funded.

The US research evaluation is fully supported by law. The functions, rights, and responsibilities of the Congress science and technology evaluation mechanism is established by the law; there are various evaluation methods, and the system in which the sponsors and operators are independent from each other effectively ensures the fairness and rationality of the evaluation. More importantly, as taxpayers, the citizens can easily and quickly query public funding conditions and yields and evaluate the performance of government-supported research activities.

In recent years, the developed countries represented by the US, the UK, Germany, and Japan have established evaluation and incentive systems based on research quality and conversion efficiency of achievement and took the conversion degree of research achievements and influence as evaluation criteria.

\subsection{Utilize information platform and big data to achieve reasonable allocation and sharing of science and technology resources to promote efficient conversion of achievements}

Developed countries make full use of information technology to achieve rational allocation, management, and sharing of science and technology resources, galvanizing the rapid conversion of research results. In 2012, the US MD Anderson Cancer Center launched the Moon Shots Program, an established data analysis platform that includes massive data on cancer patients and a series of big data analysis tools; these project resources do not belong to any team or individual but are shared by the entire Cancer Center, greatly improving the investment return and disease diagnosis. The UK Clinical Research Collaboration and Canadian Network of Networks have established a powerful information system through the unified databases, data, and operating standards and have achieved data sharing, improving the utilization of science and technology resources.

In addition, medical research has entered the "big data" era. Through the exchange, integration, and analysis of big data, new knowledge and new laws are constantly discovered, and new definitions and new value are continuously produced and created. After years of hard work, the International Cancer Ge- nome Consortium, founded in 2007, has built and consolidated a 47-country database of cancer statistics data. By comparing the genomes of normal and cancerous cells, the scientists quickly found four types of liver cancer genes and associated these genes with the corresponding treatment solutions in a relatively short period. In 2014, the US government launched the program titled "Big Data to Knowledge," aiming to enhance the efficiency and capability of using biomedical big data for the US, thus driving the relevant research and industries.

\section{Status and problems of China's medical research system building}

After more than 60 years since the founding of the Republic, China has gradually established a relatively stable and clearly structured medical research and innovation system along with the establishment of a science and technology system and the deepening of science and technology system reform. Through the implementation of a series of major medical programs such as the Technology Breakthrough Program, 863 Program, 973 Program, and National Natural Science Foundation, China's medical science and technology has continued to grow fast, greatly improving the medical research capability and science and technology level, sending the research achievements to the forefront of the world, and hugely boosting disease diagnosis and treatment as well as the biopharmaceutical industry. However, faced with the national health goal and the growing demand for medical services, China's medical research still displays weaknesses like poor innovative capability, low quality of research achievements, poor pharmaceutical R\&D capability, and so on.

\subsection{Unified planning structure still underway with a serious} shortage in medical research funding

For a long time, China's medical and health program has mainly been funded by the Ministry of Science and Technology, National Natural Science Foundation of China, and National Health and Family Planning Commission. Since the funds are from various sources, on one hand, the national medical research lacks unified planning and a subsidy system, causing a diluting or repeated subsidy or a subsidy not flowing to important research areas; on the other hand, the fixed funding for medical research institutions is inadequate, so it cannot provide longterm and stable support for the research and diagnosis of major diseases. Besides, the funding is inadequate to support scientists for free research according to their existing achievements.

Biomedical research has always been a very important field in developed countries. In 2000, the US government funded the NIH with 17.8 billion USD while the Department of Energy and National Aeronautics Space Administration received only 4 billion USD and the National Science Foundation (NSF) only 
2.7 billion USD [7]. In the past decade, the NIH budget has been as high as 30 billion USD (Fig. 1), always accounting for about $25 \%$ of the total research budget, second only to the national defense research budget. The large NIH budget reflects the important position of medical science in US society, and the achievements coming from the NIH-funded research projects have greatly improved the health of the American people and helped US medical science reach the leading position in the world.

Compared with the European and North American countries, China's medical research budget is in serious shortage. Comparing the GDP and government budget of medical research for China and the US, the UK, and Australia in 2013, the US medical research budget weight in the GDP is about 13.3 times that of China, and the UK and Australia are 4 times the size (Table 1) $[8,9]$. Considering that China has a much larger population, the gap will be bigger per capita.

The government has no overall planning and design; the research funding from different government departments are independent of each other, and the funding information cannot be shared. In addition, the research programs mainly care about the fund requirement in the certain stage but pay less attention to the conversion of research achievements; as a result, the research cannot be supported in a constant manner, and this phenomenon has become a big obstacle hindering medical innovation.

\subsection{Evaluation system of medical research fiercely criticized, urgently requiring improvement}

Science and technology evaluation plays an important part in stimulating and guiding science and technology innovation, improving governments' administration of science and technology, and building an innovative country. In recent years, catering to the quantitative evaluation, there is a widespread phenomenon of medical research institutes regarding the research evaluation as a research quantitative task. The award hierarchy, number of papers, and research budget amount have been the core indexes of professional qualification evaluation and position run.

It is very common for medical research institutes to only be concerned about the Science Citation Index (SCI) and to think highly of papers and achievements but look down on practice

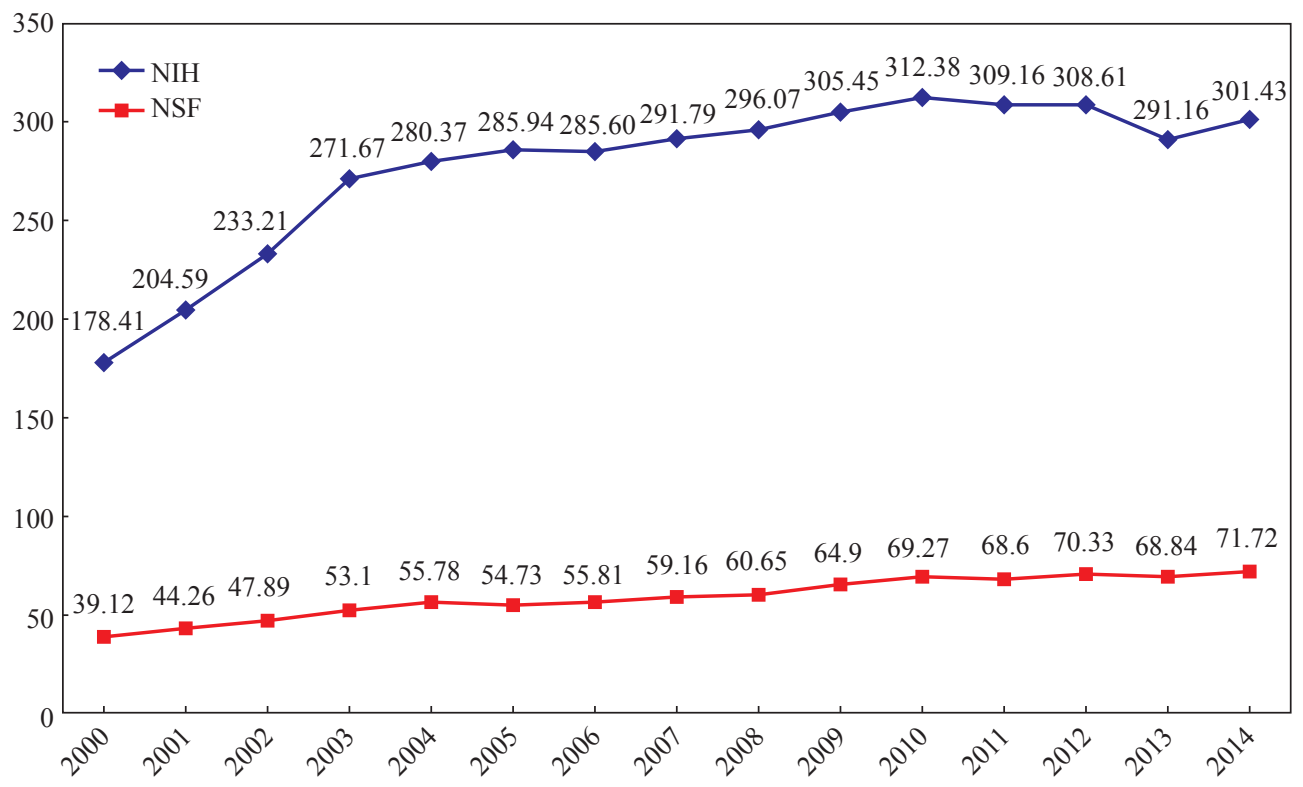

Fig. 1. Funding information of the US NIH and NSF from 2000 to 2014 (100 million USD).

Table 1. The GDP and medical research budget of four countries in 2013.

\begin{tabular}{|c|c|c|c|c|c|}
\hline & China & Australia & UK & US & $\mathrm{CN}: \mathrm{AU}: \mathrm{UK}: \mathrm{US}$ \\
\hline Total GDP ${ }^{\dagger}$ & $\begin{array}{l}9494.6 \text { billion USD } \\
(58801.9 \text { billion } \mathrm{CNY})\end{array}$ & 1560 billion USD & 2520 billion USD & 16803 billion USD & $1: 0.16: 0.27: 1.80$ \\
\hline $\begin{array}{l}\text { Weight of medical } \\
\text { research budget in GDP }\end{array}$ & $0.13 \%$ & $0.55 \%$ & $0.48 \%$ & $1.73 \%$ & $1: 4.23: 3.69: 13.31$ \\
\hline
\end{tabular}

${ }^{\dagger}$ The total GDP of China is from the National Bureau of Statistics, and those of Australia, the UK, and the US are from the World Bank. Regarding the government medical research budget, China' s fund refers to the 973 Program, 863 Program, National Science and Technology Support Program, International Cooperation Project, National Natural Science Foundation, and nonprofit industry research special funds, and so on; the US refers to the NIH annual budget; the UK refers to the MRC annual budget; and Australia refers to the NHMRC annual budget. 
and achievement conversion. Thus, China's medical research papers are expansive, but the overall quality is poor and unable to solve the practical problems. The industry still worships outside experts and science and technology and likes to imitate and copy studies; very few original technophiles are high-quality, badly affecting the R\&D of biomedicine. While 313000 of China's medical science and technology papers were recorded by the well-known database Thomson InCites during 2005-2014, ranking No. 5 in the world, the total citations only rank No. 10, and the cited frequency per paper is 10.62, ranking after Korea and the same as Brazil (Table 2) [10].

Patent is the major indicator to evaluate R\&D output. In recent years, China's medical patent application quantities grew steadily and surpassed the US to become No. 1 in the world in 2011. However, there are very few high-quality patents recognized in the three largest markets in the world. According to the Organisation for Economic Cooperation and Development database, from 2000 to 2008, only 30 of China's international patents were recognized in the three biggest markets, accounting for $1 / 50$ of the US, 1/20 of Japan, and 1/10 of Germany.

In the biomedical industry, less than $3 \%$ of the total homemade drugs in China are proprietary drugs; the rest are generic drugs. No Chinese drugs can be seen in the global top 20 best-selling dugs during 2011-2013; even worse, more than $90 \%$ of high-end medical equipment is monopolized by multinationals.

The current evaluation system in China is not complete and sound, and the evaluation indicators are too simple, unable to appropriately guide professionals to immerse themselves in research and, thus, seriously suppressing the improvement of China's overall medical science and technology.

\subsection{High barriers lead to low sharing of medical science and technology resources}

The application of science and technology resources reflects the level of a country's administration capability in this aspect, hindering the construction of a technological innovation system and sustainable socioeconomic development. In recent years, China has actively explored the solutions on the integration of science and technology resources and the sharing of scientific data, literature, and large scientific instruments and equipment. In 2000, the Ministry of Science and Technology mapped out an overall strategy to push nonprofit sharing of national scientific data and build a national sharing service system for such data. In 2010, the scientific data-sharing project - the National Population and Health Sciences Data Sharing Platform - was launched, providing sharing services for the health industry and innovative talent training.

Although the relevant parties have unanimously realized the importance of sharing science and technology resources in recent years, there are no laws to protect the sharing, resulting in high barriers to the sharing of such resources. The existing regulations are mostly administrative regulations and articles of association of respective government authorities, characterized by simplicity and brevity and not easy to apply in practice. These regulations and documents do not provide details on how to share or how the information provider can be rewarded [11]. At the same time, the current policies and legislation on science and technology resources focus on the protection of the shared information but do not provide a solution on the use of sharing information.

Since there is no overall design for the medical science and technology resources and macro coordination and administration,

Table 2. Some countries' medical research papers during 2005-2014.

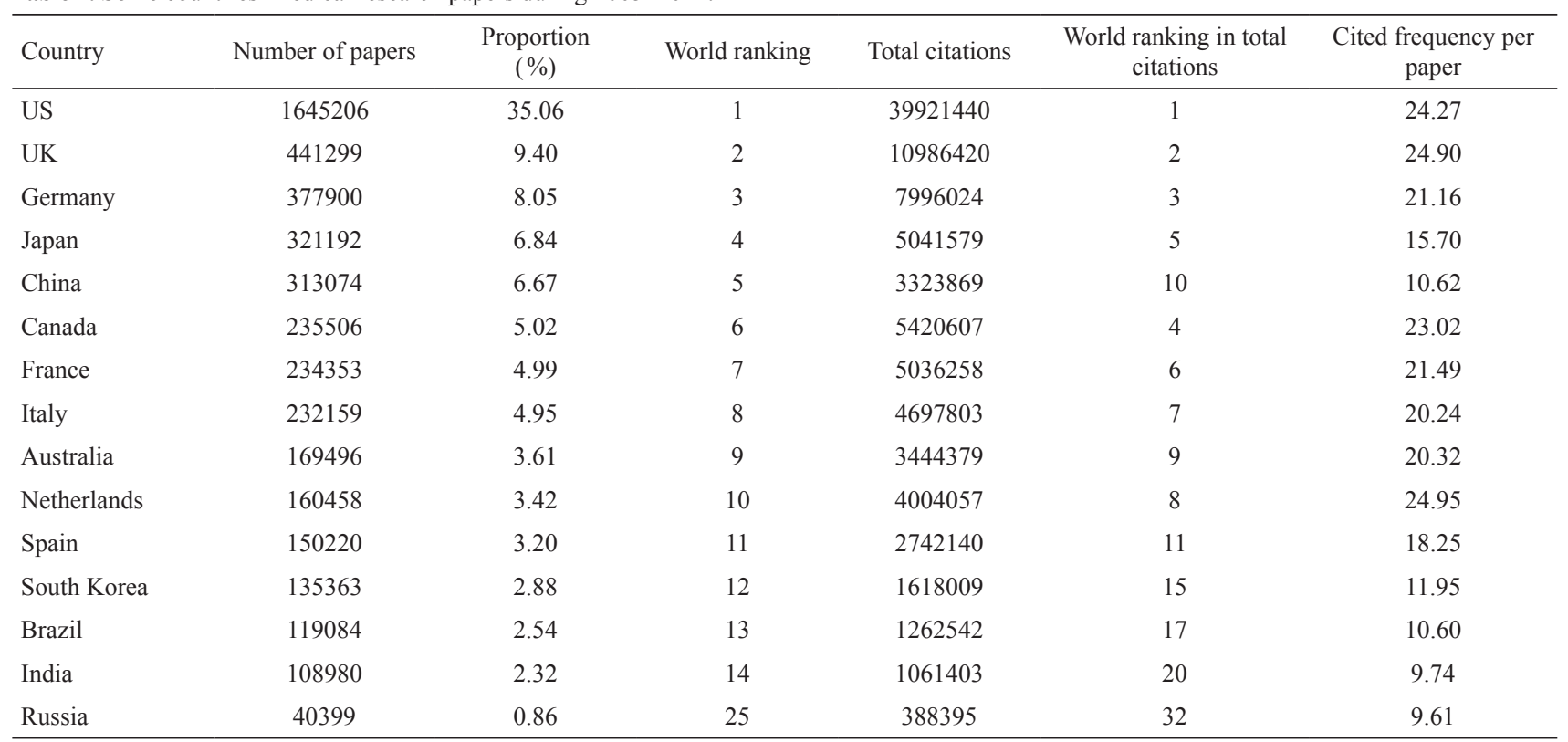


such resources are duplicated, scattered, and wasteful, unable to form the power of concentration and negatively affecting medical innovation. All relevant parties prefer the "small but complete" and "self-sufficient" mode when building medical resources, and their resources are designed for internal use instead of sharing with third parties after the completion of the resources, making the resources in each organization an isolated information island.

\section{Suggestions}

\subsection{Increase government funding to prioritize the state of medical research}

The promotion of national health and the building of "Healthy China" require the joint efforts of all stakeholders in society as well as the support of adequate resources. As China is facing a significant change in the disease spectrum and population aging and the social problems arising therefrom, the government must dramatically increase the medical research budget to guide society to pay more attention to the development of medical science and technology. To gradually increase the weight of the medical segment of the total government funding in science and technology, the funding in medical science and technology should not be below $30 \%$ of total science funding to ensure that the growth of medical innovation, medical talents, and industry development is higher than the average level of developed countries. Thus, the gap with developed countries gradually shrinks, and the utilization efficiency of funding increases, finally sending China to the position of global medical and health power in a relatively short period.

\subsection{Optimize the overall design to build a comprehensive medical research system}

To promote the growth of China's medical industry, we should first overcome the obstacles that badly hinder the development of medical research such as multiparty planning and scattered and duplicated medical funding. We should also set up a national health promotion regulator - a Ministry of Population and Health - and it should have a subsidiary — a National Health and Medical Research Institute-that will strictly follow the national long-term development plan to steer the national medical research, including developing administration policies and a strategic plan for national biomedical research and medical science and technology and coordinating the relationship between medical science and technology and medical services. In the aspect of operational mechanism, we should study the experience of the NIH, in which a small part of the research is digested by its own research institutions and the largest part of research is outsourced to third-party research institutes and individuals nationwide through competitive tender. In addition, we should strengthen the building of a medical science and technology platform to build a comprehensive medical research innovation system that includes medical science and technology authorities, research institutes, universities, medical institutions, pharmaceutical companies, and so on.

\subsection{Improve the evaluation system of medical research}

The current problems, such as the "research quantitative task" and being "only concerned about SCI," must be completely eradicated. To do this, we should fully study valuable experiences at home and abroad and in different industries and follow the $I m$ plementation Plan of Deepening Science and Technology System Reform to accelerate the development, update, and improvement of relevant rules and policies; carry out the classified evaluation system of medical professionals; and set up a capability- and contribution-based evaluation and incentive system to stimulate the healthy development of medical research and administration. In addition, we should pay more attention to the completeness and continuity of the evaluation system and continue to build an appropriate and practical evaluation method. We should also pay attention to the comprehensive evaluation of the project and relevant personnel-for example, the evaluation of the peers of the professional or research achievements, teamwork and leadership, talent training, and so on. We should gradually establish a sound evaluation, reward, and incentive system to better boost high-quality research output and achievement conversion. Additionally, the government should also develop policies and regulations to stimulate research cooperation and achievement conversion to effectively improve the innovation motivation and accelerate the quick conversion of medical research achievements.

4.4 Simultaneously focus on system building and technology application, integrate sharing resources, and promote the achievement conversion

We desperately need to use effective means to activate more science and technology resources. For this purpose, the government should develop laws, regulations, and conventions related to science and technology resources-sharing in addition to the protection of such resources, and we should also attach great importance to the sharing and utilization of science and technology resources and develop implementation measures to promote the sharing of medical resources to the utmost extent In addition, we should make the most of information technology and network technology to build a national medical research information network platform that integrates research institutes/ personnel coordination, science and technology resources, study objects, and research achievements to achieve effective integration and appropriate allocation of medical resources and promote medical innovation. 


\section{Conclusions}

Currently, "Healthy China" has been a national strategy. The 5th Plenary Session of the 18th Central Committee of the Communist Party of China proposed the grand goal to "promote the building of Healthy China," highlighting the country's determination to promote Chinese health. Strengthening a national innovation system of medical research and improving medical research capability and science and technology levels will greatly promote the realization of national health and the "Healthy China" strategy.

\section{References}

[1] NIH: Federal funds for health R\&D 2014 [EB/OL]. (2015-0203) [2016-09-25]. http://report.nih.gov/UploadDocs/T301\%20 Fed \%20Oblig \%20for \%20Health \%20RD \%20by \%20Agency $\% 202014$.

[2] Jia X F, Chen J, Zhang X Y. Study on building of national medical research system (2015 annual report of medical science and technology development in China) [M]. Beijing: China Science Publishing \& Media Ltd., 2015. Chinese.

[3] Lin H, Zhang K. INSERM functioning and implication of establishing Shanghai medical institute [J]. Chinese Health Resources, 2012.15 (4): 355-358. Chinese
[4] Zhu Q P, Qian W Q. Analysis on NIH's evaluation system of science and technology [J]. China Basic Science, 2014 (3): 33-36. Chinese.

[5] Zhou L. Supporting and management of NIH medical science foundation [J]. World Sci-tech R\&D, 2005, 27 (1): 93-99. Chinese.

[6] Gao B H, Cao J W. Evolution and enlightenment of the US NIH to China's medical research system [J]. Chinese Journal of Medical Science Research Management, 2009, 22 (5): 305-320. Chinese.

[7] Liu R. Study the mode of the US NIH to strengthen China's medical and health research system building [J]. Chinese Journal of Medical Science Research Management, 2004,17 (4): 250-252. Chinese.

[8] NHMRC. National health and medical research council annual report 2013-2014 [R]. Canberra: NHMRC, 2014.

[9] MRC. Medical research council annual report and accounts 20132014 [R]. London: MRC, 2014.

[10] An X Y, Shan L H, Gong X C. Medical research literature analysis based on incites database (2016 annual report of medical science and technology development in China) [M]. Beijing: Science Press, 2016: 363-370. Chinese.

[11] Wang Y H, Dong C, Peng J. Analysis and enlightenment of foreign natural technology resources sharing policies and regulations to China [J]. Forum on Science and Technology in China, 2008 (6) 125-129. Chinese. 